\title{
Article
}

\section{"It's hard for me to tell my story" The experiences of Aboriginal and Torres Strait Islander male clients at a residential drug and alcohol rehabilitation centre using primary health care}

Farnbach, Sara, Fernando, Jamie, Coyte, Joe, Simms, Matthew and Hackett, Maree

Available at http://clok.uclan.ac.uk/36283/

Farnbach, Sara, Fernando, Jamie, Coyte, Joe, Simms, Matthew and Hackett, Maree ORCID: 0000-0003-1211-9087 (2021) "It's hard for me to tell my story" The experiences of Aboriginal and Torres Strait Islander male clients at a residential drug and alcohol rehabilitation centre using primary health care. Health Promotion Journal of Australia, 32 (S2). pp. 87-94.

It is advisable to refer to the publisher's version if you intend to cite from the work. http://dx.doi.org/10.1002/hpja.452

For more information about UCLan's research in this area go to http://www.uclan.ac.uk/researchgroups/ and search for < name of research Group>.

For information about Research generally at UCLan please go to http://www.uclan.ac.uk/research/

All outputs in CLoK are protected by Intellectual Property Rights law, including Copyright law. Copyright, IPR and Moral Rights for the works on this site are retained by the individual authors and/or other copyright owners. Terms and conditions for use of this material are defined in the policies page. 


\section{Abstract}

\section{Issue addressed}

Aboriginal males who use drug and alcohol may experience unique barriers accessing primary health care. This study explores the perceptions of Aboriginal males in treatment for drug and alcohol use around their experiences accessing primary health care, and barriers to access.

\section{Methods}

Twenty male Aboriginal clients at a fee-paying residential drug and alcohol rehabilitation centre completed semi-structured interviews about their primary healthcare experiences before their stay. Interpretative Phenomenological Analysis was used to inductively develop themes.

\section{Results}

About half the males had regular General Practitioners at a mainstream primary health care service or Aboriginal Medical Service. Positive experiences included having medical needs met or understanding the health information provided; and negative experiences included inefficient health service or system processes or experiencing cultural bias or racism. Barriers included limited access to appointments or to the same GP regularly, long wait times, lack of access to transport, worry or fear about their health or the visit, or their complex lives taking priority.

\section{Conclusion}

This research showed that the participants sought out health care and identified barriers to accessing care and potential improvements

\section{So what?}

Access to a regular General Practitioner, continuity of care, and culturally-appropriate and comprehensive communication techniques are important to facilitate access to primary health care by Aboriginal males. Efforts to enhance access may focus on inherent strengths within Aboriginal communities including focusing on relationships between clinicians and families, providing a welcoming environment and encouraging clients to bring a trusted family member to appointments. 


\section{Introduction}

Before colonisation, Aboriginal and Torres Strait Islander people managed health and wellbeing in their communities via sophisticated farming, environmental and social systems, which provided a healthy environment for current and future generations. Colonisation ${ }^{1,2}$ and its consequences such as intergenerational trauma ${ }^{3,4}$, has led to disproportionate negative impacts to Aboriginal and Torres Strait Islander people across multiple dimensions, including a greater burden of mental illness, diabetes, cardiovascular disease and disability among some people. ${ }^{5}$ Primary health care (PHC) services are generally the first point of contact with the health system and often have a key role in screening, diagnosing, managing and coordinating care for these and other health conditions. ${ }^{6}$ Equitable access to these services is crucial to enable people to increase control over and improve their health. ${ }^{7}$ As such, the Australian Government has identified equitable access to comprehensive PHC as a focus area for addressing their priority of improving outcomes among Aboriginal and Torres Strait Islander communities. ${ }^{8}$ For PHC to be effective, it needs to be available, accessible and affordable, and clients need to feel comfortable discussing concerns about their health. ${ }^{6}$

In Australia, PHC is a community-based service delivered by General Practitioners (GP), allied health, Aboriginal health practitioners, nursing and other allied health staff. With the specific aim of providing culturally-appropriate holistic care to Aboriginal and Torres Strait Islander people, $\mathrm{PHC}$ is also delivered via a network of Aboriginal Community Controlled Health Services (ACCHS) and Aboriginal Medical Services (AMS). ${ }^{6,9}$ Unlike many PHC services which operate on a fee for service (where clients pay the gap between government subsidy), AMS clients generally have no out of pocket expenses. Although PHC should be available to all individuals, ${ }^{10}$ access to effective PHC can vary, depending on a variety of factors such as appointment availability, ${ }^{11}$ distance to travel between homes and services ${ }^{6}$ and high out-of-pocket costs. ${ }^{12}$ For instance, in very remote areas, out-of-pocket expenses are higher than in urban centres, and between $4 \%^{12}$ and $16 \%^{11}$ of Australians report not accessing PHC due to cost. Similarly, 27\% of adults reported that limited out-of-hours appointment availability was a barrier to attendance. ${ }^{11}$

For some Aboriginal and Torres Strait Islander people, there may be specific barriers to accessing PHC, such as lack of culturally-acceptable services or experiences with racism or discrimination during previous visits, making them unlikely to return. ${ }^{13}$ Males access PHC at 
lower frequencies than women, ${ }^{14}$ with Aboriginal males accessing PHC at particularly low frequencies. ${ }^{15}$ The limited available evidence around preferences for accessing health services by Aboriginal and Torres Strait Islander males identifies illness-related stigma, feelings of shame when medical jargon or terminology is used, transport barriers, financial barriers, and conflicting appointment times with other priorities as reducing the likelihood of males accessing services. ${ }^{16,17}$ In addition, the lack of access to male health staff (when this may be preferred by some Aboriginal and Torres Strait Islander males $)^{17}$, low prioritisation of preventative health, low self-esteem and low confidence may also limit access. ${ }^{16}$

This research topic was suggested by one author (Aboriginal GP working in a residential rehabilitation service) who noted that he seldom saw Aboriginal and Torres Strait Islander males seeking help for any aspect of their health from PHC services where he has worked. He questioned how frequently Aboriginal and Torres Strait Islander males' first presentation for help with drug and alcohol problems was at residential rehabilitation. The authors identified limited published research on this topic with this population. Furthermore, the experiences of Aboriginal males who use drugs or alcohol before attending a residential rehabilitation service may be different from other Aboriginal males. We explored Aboriginal males' experience of accessing PHC before attending residential rehabilitation services.

\section{Aims}

To explore the experiences of Aboriginal males who were clients at a residential drug and alcohol rehabilitation centre in New South Wales about:

1. Their experiences accessing PHC for any medical reason

2. Their reasons for non-attendance/barriers to accessing PHC when they thought they needed to see a doctor for any medical reason

\section{Methods}

\section{Approach}

This qualitative study used an Interpretative Phenomenological Analytic (IPA) approach. ${ }^{18}$ Semi-structured interviews were conducted using an interview guide.

\section{Setting and sampling strategy}

Interviews were completed with residents a fee-paying male only drug and alcohol rehabilitation centre on the Central Coast of New South Wales, Australia, between July 2017 and December 2018. The service provides a 12-week residential program that is based on 
Aboriginal values and spirituality. The program includes group counselling, one to one counselling, self-help groups and work programs. Clients also attend outside self-help meetings.

Participants were male adults who self-identified as Aboriginal or Torres Strait Islander. Clients who met the eligibility criteria were invited to participate by male Aboriginal drug and alcohol counsellors who had existing relationships with clients. The counsellors discussed the research (including the research background, aims and processes) with these clients and provided them with participant information sheets. If interested in taking part, a time was made for the potential participant to meet with the interviewers to discuss the research in detail, jointly review the participant information sheet, complete informed consent and the research interview (if consent was subsequently completed). Consenting participants were reimbursed for their time with a $\$ 25$ food voucher for a local supermarket.

\section{Data collection techniques}

Informed consent and interviews were completed by one or both male Aboriginal interviewers using a semi-structured interview guide (available on request) which included questions that addressed our two aims. The guide was pilot tested before the first interview. Interviews were digitally recorded and transcribed verbatim.

\section{Analysis}

IPA $^{18}$ can be used to examine and understand the meaning of the personal lived experiences of a situation to individuals. It is focused on an individual's personal perception or account of an event, rather than attempting to produce an objective statement of the event or using preexisting theoretical preconceptions. In order to understand the meaning of the experiences accessing PHC to the Aboriginal males, analysis followed IPA approaches. ${ }^{18}$ Aboriginal researchers were closely involved with the research to ensure cultural perspectives were central to all stages of the research including analysis. An Aboriginal Advisory Group was convened which included staff and representatives from the residential service to oversee the project and gain an in-depth understanding of the meaning of the data. All transcripts were coded by one author. Two transcripts were independently coded by a second coder, who is an Aboriginal GP. The two sets of codes were then reviewed (by the two researchers) resulting in the codes used for the remaining transcripts. The coding by the second coder provided a clinical and cultural perspectives to the analysis which were incorporated when coding the remaining transcripts. Data were managed using NVivo software. ${ }^{19}$ Twice during the 
research, the research team met to discuss progress, the developing themes and their meanings. The main themes were presented to and discussed with the Advisory Group twice (after stage one and at the end of the data collection). Memos (digital notes saved in the software) were used to record thoughts about themes as they developed and the relationship between themes that arose from discussions with the researchers and the Advisory Group. Based on analysis and discussions among authors and the Advisory Group about novel areas that were being frequently reported during interviews (e.g. the role of support people to participants), additional questions were added to the interview guide to include them in subsequent interviews. Inductive coding was completed to identify commonly reported themes related to the aims of the research.

\section{Reflexivity}

One author is an Aboriginal drug and alcohol counsellor who completed training in qualitative data collection, analysis and research processes (informed consent, study protocol). Another is an Aboriginal GP who completed training in qualitative data collection during his Master in Public Health. This author worked as a GP at the drug and alcohol rehabilitation centre from 2015 to 2019. During the conduct of the interviews, they worked as a GP in rural NSW. The lead author is a female registered nurse and researcher who had completed training in qualitative data collection, analysis and reporting. As a non-Aboriginal researcher and nurse her goal was to facilitate and support data collection, analysis and write up of the results. While initially she drafted some interview questions based on her understanding of the issues, these were modified by the Aboriginal interviewers and Advisory Group. She was aware that she needed to set aside her own views on interview completion timeframes and support the interviewers when they had the capacity. Input was sought from other members of the research team with extensive experience working with people in a drug and alcohol rehabilitation centre.

An Aboriginal Advisory Group was convened to oversee the research which included staff and representatives from the participating drug and alcohol service. For the purpose of this paper, AMS is used when clients reported using an AMS as a PHC service. If clients reported having a relationship with a specific GP, the term GP is used.

\section{Results}

Twenty males were invited to participate, and all agreed. All identified as Aboriginal most were from regional NSW, Sydney, Newcastle or another major area in NSW. Approximately 
245 minutes of interview data were recorded. No new themes were identified from the last three interviews, indicating data saturation was reached. Eighteen themes were developed. Participant demographic information is presented in Table 1.

Table 1: Participant demographics for Aboriginal and Torres Strait Islander residential drug and alcohol clients

\begin{tabular}{ll}
\hline Participant characteristics & $\mathbf{N}=\mathbf{2 0}$ \\
\hline Ethnicity & 20 \\
$\quad$ Aboriginal & 0 \\
$\quad$ Torres Strait Islander/other & \\
Age (average 27 years) & 8 \\
18-24 years & 7 \\
25-29 years & 2 \\
30-34 years & 2 \\
35-39 years & 1 \\
40-44 years & \\
Usual residence (Accessibility and Remoteness Index of Australia) & 12 \\
Regional & 5 \\
Sydney/Newcastle metropolitan & 2 \\
Major city other than Sydney & 1 \\
Remote & \\
Full or part time paid work in previous 12 months & 12 \\
Yes & 8 \\
No & \\
Previous number of stays at the service (excluding current stay) & 9 \\
0 & 6 \\
1 & 1 \\
2 & 1 \\
3 or more & 1 \\
Not reported &
\end{tabular}

\section{PHC attendance and reasons for attending}

Six clients recalled attending a PHC service or AMS in the last 12 months. About half had a GP who they saw regularly (as reported by participants). Regular GPs were male and female and from AMSs and mainstream PHC services. Being sick, needing a health or medical check (often when it was required for sport or for a Workcover claim) were the main reasons for visits. Those who visited when they were sick reported that they only went when they were really sick and 'had to'.

\section{Contrasting themes between clients who did and did not report having a regular GP}

Clients with and without a regular GP had similar experiences visiting PHC services, however their perspectives on these experiences were different.

Having a regular GP when I was a kid

Many clients reported having a regular GP when they were a child, and that they stopped seeing this GP when their lives became too complex or they lost motivation to maintain good health. Some clients still saw their childhood GP. 
Many clients with a regular GP, with whom a relationship was developed over multiple appointments, were able to make appointments to see them when needed. This contrasts with those without a regular GP who found it difficult to make appointments when they needed one, despite being willing to see any GP who was available. They believed making appointments would be easier if they had a regular GP.

Clients without a regular GP reported experiences where reception staff (usually at mainstream PHC services) were racist toward them or 'not on the same level' causing them to feel uncomfortable attending the service:

Some of them looked down their nose towards me as soon as I mentioned Aboriginal ... they just treat you a bit differently ... I try and avoid those practices.

22 years, not in full/part time work before stay, referral pathway \#13

Worry about getting bad news

Both groups worried about hearing bad news during a GP appointment. However, clients who had a regular GP reported that they were pleased when they attended and received good news, while clients without a regular GP spoke about missing appointments because of their worries.

\section{Positive experiences}

Positive experiences were more common amongst clients who reported having a regular GP.

\section{Having medical needs met, often by a trusted GP}

Fifteen clients spoke of positive experiences which included achieving the purpose of their visit, receiving thorough health care, hearing good news about their health (e.g. a blood test result), or having their needs met because their health subsequently improved. Of these, six clients reported trusting the GP, usually one who they knew well. For some, this GP had known them, their family and their history for a long time:

It was good 'cause I've been with the same doctor since I was five, so it was easy cause he's had our medication certificate [medical records], our history, for years.

23 years, in full/part time work before stay, medically or self referred \#12

\section{Visit prompted access to other health care services}


Sometimes GPs initiated access to other health services, including mental health services, substance use treatment, or the Close the Gap program (which linked them with funding for specialist health services or a prescription), and clients found this beneficial.

\section{Understanding health information}

Positive experiences were reported when clients understood what the GP told them. These experiences were usually enhanced when a family member also attended the appointment, because they asked the GP questions and explained the information to the client:

I sort of [felt] confused, I still get confused when I go to the doctors, because I don't understand myself. When they ask me a question, I don't understand ... I was used to me mum sitting in with me, that's why I wished my mum was in there [during a health check].

19 years, in full/part time work before stay, family/friend referred \#7

\section{Negative experiences}

Negative experiences were reported by clients without a regular GP. Three clients reported being dissatisfied or uncomfortable during a GP appointment when they perceived that not enough anaesthetic was used when they were having a wound stitched, they were held down as a child when having blood taken, or feeling uncomfortable when specimens from previous clients were left on the GP's desk.

\section{Service operational processes were inefficient or ineffective}

Inefficient or ineffective operational processes resulted in extended wait times to see a GP, or clients not receiving a phone call from a GP with their results when they had been told they would. These experiences were viewed negatively.

\section{Experiencing cultural bias or racism from reception staff}

In addition to the racism experienced at a mainstream PHC service (Theme having a relationship with a GP or PHC staff), one client recalled experiencing discrimination when he visited an AMS to book an appointment. Reception staff requested he provide paperwork demonstrating his 'proof of Aboriginality'. This request frustrated the client, because he knew the reception staff outside of the AMS and believed that tensions/politics within the community were the reason for the request, and that not all clients would be required to demonstrate 'proof of Aboriginality':

But it was very hard to use the [country town] AMS. 'Cause it's really political. Yeah, well the [family name removed] run the show there [at the AMS] and 
$\mathrm{f}^{* * *}$ en if you're not an [family name removed], you're not a black fella is how they see it.

34 years, in full/part time work before stay, self-referred \#15

\section{Reasons for non-attendance/barriers to accessing PHC when needing medical help (clients with and without a regular GP)}

Ten clients identified specific reasons why they had not attended a PHC service even when they were really sick and needed medical attention. Because these reasons were significant enough to stop them attending, they are conceptualised as barriers. A few clients reported that they experienced no barriers to attending PHC.

\section{Limited access to appointments}

About half the clients reported that no appointments were available, or the only available appointments were at times they could not attend due to work or family commitments. One client reported being unable to see a GP when:

As part of a court-order I had to see a doctor and it was a month before I could see a doctor, which I thought was odd. When I got out of jail I rang up and it was four weeks before I could get in for one appointment and then it was five weeks before I could get to see a psychologist.

28 years, in full/part time work before stay, referred through the justice system \#9 Many clients reported that AMSs had particularly limited appointment availability and this resulted in them attending a mainstream GP instead where more appointments were available.

\section{Unable to see the same GP regularly}

Not being able to see the same GP regularly was reported by some clients as a barrier to attending a PHC service. Many reported that GPs regularly moved practices or out of the area and new GPs did not appear to read their medical notes so were unaware of their history. For these clients, confiding in a new GP at each appointment was difficult:

It's just hard, because you're explaining, you've got to tell your story every single time. It's hard to confide in someone you haven't built up a relationship with ... it's hard for me to tell my story. I've dealt with my fair share of [expletive removed]. I never had it easy. 


\section{Lack of continuity between conversations}

Similarly, many clients reported that despite seeing the same GP several times, the GP did not remember their history or the discussions from the previous visit. This made them feel discouraged and was most commonly reported at mainstream PHC services:

But as for [mainstream practice], they ask you to make an appointment to come in the following week for results, and you'd sit down and they'd go, how can I help you today? Like they've never seen you before. It's like, you told me to come back! I just felt like starting from scratch every time.

43 years, in full/part time work before stay, referred through the justice system \#5

Despite seeing the same GP several times, these clients did not report that they perceived that their GP to be their regular GP.

\section{Lack of access to transport}

Although some clients reported difficulties using their preferred PHC service because they did not have access to transport, others reported that transport was not a barrier for them. Those reporting transport difficulties either used a different service (usually a mainstream service that was more convenient) or did not access PHC at all.

\section{Bad experiences stopped attendance}

The negative experiences described previously meant clients were less likely to return. Others reported feeling 'passed on' when a GP quickly referred them to an external service, when they would have preferred that the GP treated them directly:

It was like 'pass the buck on'. You go there for help and you get passed onto someone else. I suppose that's why I didn't feel I didn't get the help I needed ... he $[\mathrm{GP}]$ just referred me to a drug and alcohol service.

39 years, in full/part time work before stay, referred through the justice system \#4

This is in contrast to those clients who appreciated being linked with other services as described in theme Visit prompted access to other health care services.

\section{Worry or fear}

When some clients were sick, they were deterred from attending PHC services because they were worried that they would not understand the health information from the GP or fearful that they would receive bad news about their health: 
I kept delaying it, I'll go to the doctor tomorrow, I'll go to doctor tomorrow, or I'll then call up, book the appointment, wouldn't show up. I'll always miss my appointments 'cause I'm scared. You know scared, that fear factor.

22 years, not in full/part time work before stay, referral not reported \#13

[I] didn't care about my health. And sometimes scared just in case results come back bad.

28 years, not in full/part time work before stay, self-referred \#1

\section{Complex lives or life events taking priority}

Some moved to a new house or to a new town and consequently they did not visit a PHC service, even if they were very sick. Those with substantial family commitments indicated that these often took priority over their own health:

There have been times when I've been stuck at home with the kids or working too much and then having to do a lot of overtime at work and then getting there and the doctor's surgery's closed. So, I have to go to hospital to get seen by a doctor there.

36 years, in full/part time work before stay, family/friend referred \#2 


\section{Discussion}

This research shows that many Aboriginal male residential rehabilitation clients visited PHC shortly before their stay, with about half perceiving that they had a GP who they saw regularly, indicating that these males are interested in their health. Many clients described that their medical needs were met during appointments (especially if they had a GP who they perceived to be their regular GP), while some reported negative experiences such as racism or discrimination or painful clinical incidents (e.g. having a wound stitched).

Many clients described being unable to see the same GP regularly. Although some reported seeing one GP several times, they did not consider this their regular GP as the GP (usually at a mainstream PHC service) did not recall their medical history or previous discussions. This made them feel discouraged and unlikely to return. Echoing previous research, other barriers

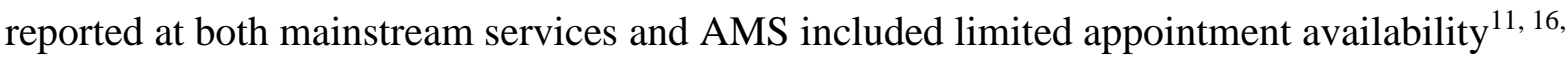
${ }^{17}$ and transport barriers ${ }^{6,16}$ which can reduce access to PHC. To the best of our knowledge the impact of limited access to an ongoing continuous GP is a new finding which highlights the importance of building relationships and continuity between clinicians and Aboriginal males. Without such continuity, we will likely miss opportunities to maximise care by intervening early around substance misuse and other disorders. These findings highlight the strengths of the different PHC service settings which may be applicable in each setting. For instance, access to mainstream PHC services may be enhanced by generating a welcoming environment by providing information/training to reception staff about speaking with Aboriginal males providing ongoing access to the same GP across multiple visits to build trust $^{21,22}$, and providing GPs with adequate time to review previous medical notes prior to each appointment. Access to AMS may be improved by making more appointments available at short notice.

This research found that Aboriginal males sometimes did not attend their appointment(s) because they worried that they would not understand the health information from the GP. Similarly, previous research has identified use of medical terminology and jargon by clinicians ${ }^{16}$ and a lack of knowledge among Aboriginal males about the different health services available (e.g. AMS and mainstream services) as impacting on their decisions, and subsequently access, to health services ${ }^{17}$ Fear of receiving bad news about their health status was highlighted as a barrier in this research, echoing concerns raised by Aboriginal males 
previously. ${ }^{17,23}$ Together, these findings indicate that there is a need for PHC staff to be able to focus on ensuring adequate time to discuss and address concerns to ensure information is understood and using culturally-appropriate communication techniques (that minimise jargon). These efforts may include the employment of skilled Aboriginal staff, a focus on understanding local community, history, culture, obligations, strengths and needs, and a flexible approach to care. The values of Aboriginal people and may be formally acknowledged and supported with existing or locally developed protocols ${ }^{24}$. Policymakers need to consult with the broadest range of PHC stakeholders to improve the legitimacy of policies. The establishment of community advisory boards will allow for community to better advocate for their own needs. The impact of these initiatives should be formally evaluated. ${ }^{25}$ The positive role of families in supporting the Aboriginal males to access health care is also apparent, both by linking them with PHC services (through an established relationship with a GP) and by attending appointment to support them during discussions with health staff. Other enablers identified include the existence of trust of PHC by communities and families ${ }^{21}$, a physically and emotionally welcoming environment ${ }^{21,22}$ and bringing a trusted family member to appointments. Providing comprehensive health care (that responds to holistic health needs) may also be important when accessing PHC. ${ }^{22}$ Holistic approaches such as these should be planned in conjunction with local Aboriginal representatives and could be modelled on those used by AMSs.

PHC staff should consider the meaning that tests may have for their clients and how results might impact on their health, social situation and their relationship with the justice system. This may enhance the experiences of Aboriginal males when visiting PHC, encouraging ongoing contact with PHC and overcoming these barriers.

Similarly to previous research, ${ }^{17,26}$ this group of Aboriginal males were interested in their health and sought support for their health, which is in contrast to some misconceptions held about Aboriginal males which suggest ${ }^{26}$ that a lack of motivation or interest stops them from accessing health care. We have identified opportunities to strengthen PHC service provision (so males have access to a regular GP) and approaches (to enhance communication of health information) to better support males, in order to improve health outcomes. Referring on to an external service should be discussed with, rather than imposed on, clients to determine how such suggestions may be interpreted and whether or not they would be welcome. 


\section{Strengths and limitations}

Asking participants to recall unpleasant events from a long time ago relies on clients remembering these events clearly, potentially leading to some recall bias in this research. The existing relationship between clients and the interviewers may have enhanced participation in this study and encouraged open and honest conversations. However, these relationships may have also caused clients to over-report the frequency of contact with PHC. However, we believe this approach facilitated the research (all males who were asked took part), which provided new evidence in an area where limited research has been done. All participants were from one residential rehabilitation centre in NSW with only one from regional NSW, therefore this research may not represent all Aboriginal males in treatment for drug and alcohol use.

\section{Conclusions}

Many of the barriers identified, such as limited appointment availability and lack of continuity between staff and appointments, highlight potential opportunities to enhance PHC and improve access for Aboriginal males in treatment for drug and alcohol use. There is a need for the provision of individualised and culturally appropriate continuity of care if Australia is to achieve equitable access to comprehensive PHC among Aboriginal communities. ${ }^{8}$

\section{Conflict of interest}

The authors declare no conflicts of interest.

\section{References}

1. Calma T, Dudgeon P, Bray A. Aboriginal and Torres Strait Islander Social and Emotional Wellbeing and Mental Health. Australian Psychologist. 2017;52(4):255-60.

2. Sherwood J. Colonisation-It's bad for your health: The context of Aboriginal health. Contemporary nurse. 2013;46(1):28-40.

3. Testro P, Ryan K, Hillan L. Restoring our spirits - reshaping our futures creating a trauma aware, healing informed response to the impacts of institutional child sexual abuse for Aboriginal and Torres Strait Islander people. Canberra: The Healing Foundation; 2016.

4. Atkinson J. Trauma trails, recreating song lines: The transgenerational effects of trauma in Indigenous Australians. North Melbourne: Spinifex Press; 2002.

5. Australian Institute of Health and Welfare. The health and welfare of Australia's Aboriginal and Torres Strait Islander peoples (Ch 5). Canberra; 2015. 
6. Australian Institute of Health and Welfare. 6.6 Indigenous Australians' access to health services. Canberra; 2016.

7. World Health Organization. The Ottawa Charter for Health Promotion. Geneva, Switzerland: WHO; 1986, Nov 21 [Available from: http://www.who.int/healthpromotion/conferences/previous/ottawa/en/index.html.

8. Health Do. Australia's long term national health plan to build the world's best health system. Canberra: Australian Government; 2019.

9. Central Australian Aboriginal Congress. Social and Emotional Wellbeing Service Alice Springs 2016 [Internet] [Available from: http://www.caac.org.au/client-services/socialemotional-wellbeing-service.

10. International Conference on Primary Health Care. Declaration of Alma-Ata USSR, 1978 [Available from: https://www.who.int/publications/almaata_declaration_en.pdf.

11. Corscadden L, Levesque J-F, Lewis V, Breton M, Sutherland K, Weenink J-W, et al. Barriers to accessing primary health care: comparing Australian experiences internationally. Australian journal of primary health. 2017;23(3):223-8.

12. Swerissen H, Duckett S, Moran G. Mapping primary care in Australia. Grattan Institute; 2018.

13. Davy C, Harfield S, McArthur A, Munn Z, Brown A. Access to primary health care services for Indigenous peoples: A framework synthesis. International journal for equity in health. 2016;15(1):163.

14. Welfare. AIoHa. The health of Australia's males [Internet]. . Canberra: Australian Institute of Health and Welfare,; 2019.

15. Australian Department of Health and Ageing. National Aboriginal and Torres Strait Islander Health Plan 2013-2023. Canberra (AUST): Government of Australia; 2013.

16. Wenitong M, Adams M, Holden CA. Engaging aboriginal and Torres Strait islander men in primary care settings. The Medical Journal of Australia. 2014;200(11):632-3.

17. Canuto K, Wittert G, Harfield S, Brown A. "I feel more comfortable speaking to a male": Aboriginal and Torres Strait Islander men's discourse on utilizing primary health care services. International journal for equity in health. 2018;17(1):185.

18. Smith JA. Qualitative psychology: A practical guide to research methods: Sage Publications, Inc; 2003.

19. QSR International Pty Ltd. NVivo 10 for Windows 2013 [Accessed 01/11/2015]. Available from: http://www.qsrinternational.com/product.

20. Commonwealth of Australia. Ethical conduct in research with Aboriginal and Torres Strait Islander Peoples and communities: Guidelines for researchers and stakeholders. Canberra; 2018.

21. Davy C, Cass A, Brady J, DeVries J, Fewquandie B, Ingram S, et al. Facilitating engagement through strong relationships between primary healthcare and Aboriginal and Torres Strait Islander peoples. Australian and New Zealand Journal of Public Health. 2016;40(6):535-41.

22. Gomersall JS, Gibson O, Dwyer J, O'Donnell K, Stephenson M, Carter D, et al. What Indigenous Australian clients value about primary health care: a systematic review of 
qualitative evidence. Australian and New Zealand journal of public health. 2017;41(4):41723.

23. Isaacs AN, Maybery D, Gruis H. Help seeking by Aboriginal men who are mentally unwell: a pilot study. Early intervention in psychiatry. 2013;7(4):407-13.

24. Working Together: Aboriginal and Torres Strait Islander Mental Health and Wellbeing Principles and Practice. Purdie N, Dudgeon P, Walker R, editors. Canberra: Department of Health and Aging; 2010.

25. World Health Organization. Operational framework for primary health care Transforming vision into action. Draft for Member States' consideration at the

Seventy-third World Health Assembly 2020 [17/12/2020]. Available from:

https://www.who.int/servicedeliverysafety/operational-framework-for-primary-healthcare WHA73.pdf.

26. Smith JA, Braunack-Mayer A, Wittert G, Warin M. "It's sort of like being a detective": Understanding how Australian men self-monitor their health prior to seeking help. BMC Health Services Research. 2008;8(1):56. 